\title{
Risk management of vaginal birth after cesarean section (Review)
}

\author{
ALEXANDRU CARAULEANU, INGRID ANDRADA TANASA, DRAGOS NEMESCU and DEMETRA SOCOLOV
}

Department of Obstetrics and Gynecology, 'Grigore T. Popa' University of Medicine and Pharmacy, 700115 Iasi, Romania

Received May 28, 2021; Accepted June 29, 2021

DOI: $10.3892 / \mathrm{etm} .2021 .10545$

\begin{abstract}
The increasing number of patients who desire to experience vaginal birth after cesarean (VBAC) and the optimized protocols for trial of labor after cesarean (TOLAC) has led to a shift of old obstetrical paradigms. The VBAC trend is accompanied with numerous challenges for healthcare professionals, from establishing suitability of each pregnant patient profile for TOLAC to active labor management, and ethical or legal issues, which occasionally are not included in specific guidelines. That is why an individualized risk assessment and management can serve obstetricians as a useful tool for improving outcomes of patients, satisfaction, and also for avoiding legal or moral liabilities. The risk management concept aims to reduce foreseen risks and to emulate strategies for prediction and prevention of unwanted events. In obstetrics, and particularly for the VBAC topic, this concept is relatively new and undefined, and thus its features are disparate between guideline recommendations and clinical studies. This narrative review intends to offer a new and organic perspective over clinical aspects of TOLAC and VBAC risk management.
\end{abstract}

\section{Contents}

1. Introduction

2. Materials and methods

3. Risk factor identification

4. Risk factor analysis

Correspondence to: Dr Ingrid-Andrada Tanasa, Department of Obstetrics and Gynecology, 'Grigore T. Popa' University of Medicine and Pharmacy, 16 Universitatii Street, 700115 Iasi, Romania

E-mail: tanasaingrid@yahoo.com

Abbreviations: ACOG, American College of Obstetricians and Gynecologists; AUC, area under the curve; BMI, body mass index; CS, cesarean section; ECV, external cephalic version; EFW, estimated fetal weight; ERCD, elective repeat cesarean delivery; NCHS, National Center for Health Statistics; PPCS, planned primary cesarean section; $\mathrm{PGE}_{1}$, misoprostol; SOGC, Society of Obstetricians and Gynaecologists of Canada; TOLAC, trial of labor after cesarean; VBAC, vaginal birth after cesarean

Key words: TOLAC, VBAC, risk assessment, risk management, cesarean section
5. Risk factor ranking

6. Risk factors management and monitoring

7. Conclusions

\section{Introduction}

Successful trial of labor after cesarean (TOLAC), followed by vaginal birth after cesarean (VBAC) are two desiderates of modern obstetrical health policies worldwide, and developed countries are investing more in strategies that ensure safety of patients in a controlled hospital environment, with reduced costs.

Recent data published by the National Center for Health Statistics (NCHS) in March 2020 outlined the increasing trend of VBAC in the United States of America (USA) from $12.4 \%$ in 2016 to $12.8 \%$ in 2017 and $13.3 \%$ in 2018 (1). Moreover, this ascending trend was observed for an age range between 20-30 years, and for a gestational age of 38 weeks and over (1). This data reinforces the idea of an obstetrical phenomenon which arose from the need of respecting the autonomy of patients, while its complex features reflect on different health care systems and policies.

In 2010, the National Institutes of Health recognized that TOLAC was a reasonable option for numerous women with a prior cesarean delivery, but at the same time the willingness of physicians and healthcare institutions to offer TOLAC can be influenced by concerns over liability (2).

Indeed, the legal framework and local protocols for VBAC and TOLAC are extremely heterogenous along different countries. Moreover, the risks associated with unsuccessful vaginal delivery after cesarean could be considered pretexts for malpractice lawsuits.

The American College of Obstetricians and Gynecologists (ACOG), signed in 2019, a practice bulletin that evaluated the risks and benefits of TOLAC, and provided practical guidelines for counseling and management of patients who wish to attempt VBAC. In this publication, it was recognized that VBAC is associated with lower rates of hemorrhage, thromboembolism, and infection, and a shorter recovery period compared with women who have an elective repeat cesarean delivery (ERCD). Moreover, it was acknowledged that VBAC may decrease the risk of maternal consequences related to multiple cesarean deliveries (3).

A risk is the potential of a situation or event to affect the achievement of specific objectives. Risk management refers to those processes that allow individual risk events and overall 
risk to be understood and managed proactively, in order to maximize the chances of success by enhancing positive opportunities and outcomes and reducing potential threats (4). The risk management process can be summarized into 5 simple steps: Identifying, analyzing, ranking, treating, and monitoring a specific risk.

The implementation of a large scale, institutional process of risk management is a cost-effective strategy, that implies specialized expertise and in-depth analysis of the local activity profile of the health facilities. The resulted information must reflect on a risk management strategy and be further reviewed in order to evaluate its efficacy. Moreover, after local implementations of these strategies, the information could be gathered in a big-data database, and governments, along with experts from the national and international obstetrical institutions, could evaluate them and promote an initiative that corrects the flows associated with VBAC management.

This narrative review aimed to offer a new perspective over clinical aspects of TOLAC and VBAC risk management.

\section{Materials and methods}

A literature review was carried out to identify all relevant studies that evaluated the risk management of VBAC between the 1st of January 1990 and the 31st of December 2020. The main scientific databases (PubMed, Scopus and Web of Science) were searched for the following search terms: 'VBAC'; 'risk management'; 'risk factors'; 'TOLAC', using the function 'AND' and 'OR'.

The bibliographies of all relevant articles, including reviews, were screened for further references. Only the articles written in English were evaluated. After deleting duplicates, titles, abstracts, or entire articles were further screened. Articles that solely reported data collected before 1990 were excluded in an effort to focus on current barriers. Screening was carried out independently by two authors (AC and IAT). Any disagreement concerning eligibility between reviewers was resolved by a third author (DS). A total of 50 scientific studies were included in this review.

\section{Risk factor identification}

While there are numerous factors that can influence the evolution of TOLAC and VBAC, only a couple of them may heavily tip the balance to one decision or another.

From a risk management perspective, the clinician must identify risks before adverse events occur, and put into place procedures, barriers or other measures to reduce these risks.

The process of risk identification must always consider objectives and activities mentioned in a strategic plan. Whether talking about the general and specific objectives, or about the activities that make those objectives possible, it is always important to have a systematic and multidisciplinary approach.

As for VBAC, the general objectives can be summarized as follows: i) To ensure a safe and successful VBAC; ii) to maintain an open collaboration with the patient; iii) to effectively select candidates for TOLAC; iv) to accurately assess and manage complications related with TOLAC and VBAC; v) to provide patient education and guidance throughout the prenatal and intrapartum period; vi) to establish a legal framework for healthcare professionals that choose to conduct a VBAC; vii) to implement strategies at an institutional level for optimizing the VBAC process; and viii) to develop educational and training programs for healthcare workers $(3,5)$.

The most used instrument for identifying risk factors remains patient history, which is readily available as early as the first trimester visit, followed by sonographic evaluation of the fetus [e.g., estimated fetal weight (EFW)] or the uterus (e.g., uterine scar position, thickness, placental position).

Specific objectives depend on the local decisions and may vary in different regions of the world. They may include development of local protocols for TOLAC and VBAC management, equipping the hospital with all the necessary equipment, training courses and simulations for healthcare professionals.

As for activities that support the implementation of the objectives, the following are suggested: Prenatal screening and prediction of adverse maternal and neonatal events, development of campaigns and informal sessions on various platforms for patient education, creation of a fully equipped health facility with trained personnel, development of internal and national protocols for TOLAC and VBAC, implementation of courses, simulations and training modules for healthcare professionals, creating a multidisciplinary team at a governmental level for developing long-term and sustainable strategies for reducing VBAC-related maternal and fetal morbidity and mortality.

The most favorable factors that offer a high probability (>60-70\%) of achieving VBAC are considered (3): i) Women with one previous cesarean delivery with a low-transverse incision; ii) women with a previous vaginal delivery, either before or after the cesarean delivery; iii) active labor settled at 40 weeks of gestation or less, with an EFW of $<4,000 \mathrm{~g}$ and a favorable cervix; iv) time interval since the last cesarean section $(\mathrm{CS})>1$ year; v) absence of serious maternal comorbidities (e.g., cardiovascular, renal and metabolic); and vi) Health facility with right equipment and trained personnel.

On the other hand, factors that may negatively influence the probability of achieving a VBAC include (3): i) Women with previous low vertical incision or unknown type of incision; ii) previous uterine rupture; iii) breech or transverse presentation; iv) placenta praevia or abnormally adherent placenta; v) EFW of 4,000 g or more; vi) maternal obesity [body mass index (BMI) >40]; and vii) two or more previous CSs.

All these factors should be considered when attempting a TOLAC and maternal consent should be obtained before proceeding to a specific method of delivery. Some of these factors must be correlated with the history of patients. For example, if a woman had a previous vaginal delivery of a macrosomic fetus, an EFW of $>4,000 \mathrm{~g}$ should not preclude TOLAC (3).

The next step is to identify the vulnerabilities (internal weak points) or external threats (6). Internal vulnerabilities can be considered the personnel experience and 'know-how', hospital facilities, internal problems of communication, lack of internal protocols and administrative issues. External threats refer to pressure exerted by mass-media and public opinion, frequent changes in regulations and legal framework, malpractice lawsuits, diminished budget, political uncertainties, special epidemiological situations (e.g., SARS-CoV-2 pandemic). 


\section{Risk factor analysis}

Risk factor analysis assumes the estimation of risk manifestation into real situations and is based on a probabilistic approach (7). As for the prenatal care, some algorithms have been developed to estimate the likelihood of successful TOLAC, and some of them were successfully implemented into multiple clinical settings.

In 2007, Grobman et al developed a model based on factors available at the first prenatal visit that predicted the chance of VBAC for pregnant patients who underwent TOLAC (8). The resulted predictive nomogram incorporated six variables: Maternal age, BMI, ethnicity, prior vaginal delivery, the occurrence of a VBAC, and a potentially recurrent indication for the cesarean delivery, and had an area under the curve (AUC) of 0.754 (95\% confidence interval 0.742-0.766) (8). This AUC was increased when adding delivery unit admission features up to $0.77-0.80(9,10)$. A recent study by Harris et al compared the predictive performance of these 3 prediction models (Grobman 2007, Grobman 2009, and Metz 2013) with the use of a single tertiary referral cohort and concluded that in their health system, the Grobman 2007 and Metz VBAC models were accurate when probabilities of achieving a VBAC were $>60 \%$, with predicted success rates of $60-90 \%$, but their accuracy was not reliable when predicted success rates for VBAC were $<60 \%$ (11). Evidence has indicated that women with at least a $60-70 \%$ probability of achieving a VBAC, experience the same or less maternal morbidity than women who have an $\operatorname{ERCD}(12,13)$.

In 2020, Lipschuetz et al used a machine learning algorithm to offer a personalized prediction tool for VBAC with an AUC of 0.745 (95\% CI=0.728-0.762) when using data available at the first antenatal visit and increased AUC of 0.793 (95\% CI=0.778-0.808) with the addition of delivery unit admission features (14). These data revealed at least a similar predictive power of machine learning algorithm with that of the aforementioned models, and indicated that this type of modern tool may aid patient-physician decision making.

Another tool for risk analysis is represented by various types of probability scales, that can assess quantitatively or qualitatively the effect of risks factors on the final outcome (15). More data and validation studies will be required to assess the effectiveness of these type of instruments in predicting risk materialization, but experience from other domains suggests promising results.

\section{Risk factor ranking}

By far, the most important risk associated with TOLAC is uterine rupture (3). Although there are some heterogenous definitions of uterine rupture, it implies a solution of continuity at the level of the uterine scar that affects all uterine wall layers; amniotic membranes, umbilical cord or fetal parts can extrude through this type of defect. Moreover, the uterine scar rupture can be asymptomatic, which is a challenging situation for the obstetrician in terms of recognition and management. Uterine rupture can happen during labor or during the entire pregnancy (16-18).

The risk factors that increase the chance of uterine rupture include higher maternal age, women with $>1$ previous CS, type of previous CS incision, and decreased scar myometrial thickness on ultrasound $(3,19)$.
Sonographic evaluation of uterine scar thickness can be a useful tool for prediction of uterine rupture when used in conjunction with other risk factors. In a cross-sectional validation study, Sarwar et al (20) concluded that a cut-off value of $\leq 5 \mathrm{~mm}$ of uterine scar thickness, had a sensitivity of $76.9 \%$, specificity of $48.7 \%$ and accuracy of $58.12 \%$ for prediction of uterine rupture and additional factors should be considered when choosing a mode of delivery.

If the uterine rupture risk is to be assessed from a probabilistic scale with 3 steps, based on effect and probability of manifestation into real cases, an extremely high effect on outcome of patients will be noticed, but low probability of occurring. This model can be extended to other factors, depending on the available clinical and safety data.

The indication for primary CS (PCS) must be carefully assessed, and a second opinion can be beneficial in terms of avoiding risks associated with this type of surgical intervention. Although placenta praevia remains an indication for elective CS, the actual proof of existence is occasionally omitted, especially when sonographic findings are not indicative of exact placental position and the actual organ is removed during surgery. The reasons for this grey obstetrical situation are mainly based on the need of obstetricians for justification in case of elective PCS, but in the long run, this aspect can bring unwanted psychological trauma for the mother, as well as increased costs of future births for the healthcare system.

In a population-based cross-sectional study, Cegolon et al (21) examined the patterns of PCS, planned PCS (PPCS), VBAC and associated factors. The top three determinants for PCS and PPCS in this study were breech presentation, placenta praevia/abruptio placentae/ante-partum hemorrhage and non-reassuring fetal status, while VBAC-1 was more likely with gestation $\geq 41$ weeks, placental weight $<500 \mathrm{~g}$ and labor analgesia. The VBAC-1 rate (28.4\%) in this study was almost three times the Italian national rate of $9 \%$ reported for 2017, and the authors suggested that a careful evaluation of indication for PCS as well as staff education, prenatal counseling, clinical audit and financial rewards could be beneficial in term of reducing the primary cesarean delivery rates and promoting VBAC.

In a retrospective study published in 2020 (22), the authors investigated the effect of demographic, socioeconomic, and health system factors on TOLAC and TOLAC failure in low risk pregnancies, and their variation over time. Advanced maternal age was associated with both low TOLAC rates and high TOLAC failure, while ethnicity (women from East Asia, Latin America, non-western origin), education ( $<11$ years of education) and health system factors (e.g., delivery unit size and administrative region) had a considerable effect on both TOLAC and TOLAC failure. The authors also identified a significantly stronger association between TOLAC failure and short education or small size of delivery over time, as well as a weaker association between non-TOLAC and maternal age $>39$ years. These data indicated that maternal age may be less influential over the TOLAC success rate.

\section{Risk factors management and monitoring}

After risk identification and evaluation, it is necessary to establish the type of response to each individual risk by 
establishing some management strategies tailored to specific clinical situations.

While spontaneous labor is preferred for TOLAC, different pharmacological or mechanical methods for an active management can be used in various clinical situations, especially if the gestational age exceeds 40 weeks of gestation.

Generally speaking, risk acceptance is a type of response to risk defined by the lack of control measures and is appropriate for risks that have a lower exposure than the tolerance limit. An example is VBAC in case of an EFW of $>4,000 \mathrm{~g}$ for a patient with previous low-transverse cesarean section that has delivered vaginally a macrosomic baby. In this case, it is reasonable to allow TOLAC and to monitor both mother and fetus for adverse events.

Pharmacologic agents used for cervical ripening or induction of labor include prostaglandin $\left(\mathrm{PGE}_{2}\right)$ analog, misoprostol ( $\mathrm{PGE}_{1}$ ) or mifepristone (23). Clinical guidelines agree that inappropriate induction and augmentation of labor with oxytocin increases the risk of uterine rupture for women attempting TOLAC (3). Moreover, both the ACOG and Society of Obstetricians and Gynaecologists of Canada (SOGC) recommend against misoprostol use due to the same outcome $(3,19,24)$. This last recommendation is an example of risk avoidance by eliminating a factor which may pose an increased risk of uterine rupture.

As for clinical evidence over the efficacy of different methods for labor induction and augmentation, data is scarce, mainly due to the small sample size, heterogeneity, and inconsistency of the studies.

A systematic review and meta-analysis conducted by Wingert et al (25) demonstrated some evidence of higher VBAC rates among women who underwent spontaneous labor, when compared with women whose labors were induced, regardless of method or agent used for induction.

In another systematic review, Catling-Paull et al (26) revealed that inductions of labor by amniotomy, prostaglandins, or oxytocin (or a combination of these methods) were associated with lower rates of vaginal deliveries.

A Cochrane systematic review compared women with a prior cesarean delivery undergoing cervical ripening and/or labor induction with placebo, no treatment or other methods, and revealed overall moderate to low certainty of evidence for these interventions (27).

Once labor starts, the patient attempting VBAC must be monitored by an obstetrician or other obstetric care provider in a hospital that facilitates immediate intervention in case of emergency. This is an example of active risk management by taking control measures for inherent risks that may expose the mother and fetus to severe negative events.

A continuous electronic fetal monitoring is necessary because in $70 \%$ (3) of uterine rupture cases a fetal heart rate aberrancy is manifested and urges intervention. Other signs and symptoms of uterine rupture may include fetal bradycardia, increased uterine contractions, vaginal bleeding, loss of fetal station, or new onset of intense uterine pain (28-30).

The typical manifestations of uterine rupture are acute onset abdominal pain, vaginal bleeding, a non-reassuring fetal heart rate tracing, and a change in the contraction pattern on cardiotocography $(31,32)$.
If the patient is stable, without signs of hypovolemia, an ultrasound could be performed. The sonographic markers that support the diagnosis of uterine rupture are an aberrancy in the uterine wall, a hematoma next to a hysterotomy scar, free fluid in the peritoneum, or fetal parts outside the uterus (33). Ultimately, the definite diagnosis is obtained through laparotomy with identification of the uterine defect, fetal parts, and hemoperitoneum.

In case of high suspicion of uterine rupture, an emergency cesarean section is required under general anesthesia, with a midline abdominal incision for faster access and better visualization of the peritoneal cavity. If the uterine rupture is small, the surgeon can proceed to uterine repair (34), otherwise, especially in hemodynamic instability, a hysterectomy is indicated (35).

A prenatal management of modifiable risk factors should be structured on 4 pilons: Maternal, fetal, healthcare professionals or hospitals, and government institutions.

The maternal factors that can be controlled are some maternal comorbidities (i.e., glycemic and hypertension control, stabilization of thyroid, autoimmune and infectious disorders), BMI, and indirectly maternal age by choosing to start a family earlier in life (36). Unfortunately, the last desiderate is less frequent, especially in developed countries, where young women prefer to concentrate on their career.

As for fetal factors, perhaps the most important modifiable factor is the fetal presentation. For breech presentation, external cephalic version (ECV) can be an option. Despite limited evidence (37-40), it appears that ECV is possible for women with prior low-transverse hysterotomy who are otherwise suitable candidates for TOLAC and have no contraindications for such a procedure.

Another fetal factor that must be considered is prematurity associated with small for gestational age fetuses which usually indicates cesarean section and leads to increased fetal morbidity and mortality as stated by Turcan et al, in a recent study (41). Among the important complications that are associated with this category of newborns the following must be mentioned: Cerebral edema, pulmonary hemorrhage, neonatal seizures and disseminated intravascular coagulation, persistence of the arterial canal, cerebral hemorrhage, hyaline membrane disease, and retinopathy (42). All these neonatal complications must be considered when selecting a suitable candidate for TOLAC.

The healthcare professionals represent the most important risk managers and resources that make possible the VBAC experience. However, there are numerous factors that influence the decisions and the course of action of an obstetrician when confronted with a VBAC case. Whether it is about lack of specific education, resources, experience or fear of legal liabilities and malpractice lawsuits, this category can be considered an Achilles' heel due to its vulnerability. Moreover, the commodity and limitations of some professionals are real impediments to implementing a generalized strategy for VBAC in numerous countries.

Several methods for assessing the needs and visions of healthcare professionals have been described and implemented in different healthcare facilities. These include semi-structured interviews and questionnaires, as well as brainstorming sessions and debates (43-45). However, a targeted strategy for TOLAC and VBAC remains under construction. 
Nonetheless, medicine is a partnership, and the willingness of numerous women with previous CSs to experience VBAC is becoming more and more vocal. There are numerous social media groups (46) and organizations worldwide that promote the respect for autonomy of patients and freedom of choice. While it is equally true that those groups are also platforms for misinformation, it is our duty, as healthcare professionals and partners in this wonderful journey of giving life, to advise and protect the best interests of both mother and fetus.

Moreover, the social media platforms can serve healthcare professionals as instruments for preferences of patients, views and complaints, as an Australian VBAC survey demonstrated (47). In this study the patients were pleased by the continuity of the care system, feeling more in control and respected by midwives. These aspects outline the importance of a long-term relationship based on reciprocal respect and appreciation.

In a recent systematic review and meta-analysis conducted by Poprzeczny et al (48), the use of patient decision reduced decisional conflict and improved patient knowledge. These conclusions highlight the importance of shared-decisions and communication between obstetricians and patients, a concept that can be extrapolated inclusively to the delicate topic of VBAC.

The legal background is a key element that must be developed and respected in all health care facilities. Moreover, the local and national protocols must be created and updated in conformity with the recommendations of international organizations of obstetrics and gynecology.

Finally, the governments must implement risk management strategies and methodologies for healthcare systems that are aligned with the regional visions and context.

\section{Conclusions}

VBAC is an increasing trend among various healthcare systems in the world and obstetricians need to adapt their surveillance and case-management approach to the new norms and requirements.

Anamnesis, ultrasonography, screening models and calculator are useful tools for identification of risk factors and complications associated with TOLAC or VBAC.

The mode of delivery must be carefully assessed and adopted in accordance to the autonomy and choice of patients. Moreover, the indication for the first cesarean intervention must be weighed, and indications such as placenta praevia must truly reflect its low incidence $(49,50)$.

The uterine rupture is the most important adverse effect associated with failed TOLAC (3), and an active labor monitoring strategy is required for detection of signs and symptoms that indicate such a complication.

Although risk management is a barely exploited concept in the obstetrical domain, and more specific for the TOLAC and VBAC topics, it is anticipated that further studies will reveal the importance and effectiveness of this complex process regarding the prediction, prevention and management of risks and events.

\section{Acknowledgements}

Not applicable.

\section{Funding}

No funding was received.

\section{Availability of data and materials}

Not applicable.

\section{Authors' contributions}

AC and IAT designed the study. AC drafted the initial manuscript. DN and DS carried out the reference search, supported by AC and IAT, who retrieved the evidence and selected the papers. AC, IAT and DS extracted the data; IAT, AC, DN, and DS participated in writing the final version of the manuscript. IAT and AC were assigned to perform a final check of the manuscript. All authors read and approved the final manuscript.

\section{Ethics approval and consent to participate}

Not applicable.

\section{Patient consent for publication}

Not applicable.

\section{Competing interests}

The authors declare that they have no competing interests.

\section{References}

1. Osterman MJK: Recent trends in vaginal birth after cesarean delivery: United States, 2016-2018. NCHS Data Brief 359: 1-8, 2020.

2. No authors listed: National institutes of health consensus development conference statement: Vaginal birth after cesarean: New insights March 8-10, 2010. Obstet Gynecol 115: 1279-1295, 2010.

3. ACOG practice bulletin no. 205: Vaginal birth after cesarean delivery. Obstet Gynecol 133: e110-e127, 2019.

4. Sghaier W, Hergon E and Desroches A: Global risk management. Transfus Clin Biol 22: 158-167, 2015 (In French).

5. Schifrin BS and Cohen WR: The effect of malpractice claims on the use of caesarean section. Best Pract Res Clin Obstet Gynaecol 27: 269-283, 2013.

6. Scholefield H: Safety in obstetric critical care. Best Pract Res Clin Obstet Gynaecol 22: 965-982, 2008.

7. Sambo F, Ferrazzi F and Bellazzi R: 12-probabilistic modelling with bayesian networks. In: Modelling methodology for physiology and medicine. 2nd edition. Carson E and Cobelli C (eds). Elsevier, Oxford, pp257-280, 2014.

8. Grobman WA, Lai Y, Landon MB, Spong CY, Leveno KJ, Rouse DJ, Varner MW, Moawad AH, Caritis SN, Harper M, et al: National institute of child health and human development (NICHD) maternal-fetal medicine units network (MFMU): Development of a nomogram for prediction of vaginal birth after cesarean delivery. Obstet Gynecol 109: 806-812, 2007.

9. Grobman WA,Lai Y,Landon MB, Spong CY,Leveno KJ, Rouse DJ, Varner MW, Moawad AH, Simhan HN, Harper M, et al: Does information available at admission for delivery improve prediction of vaginal birth after cesarean? Am J Perinatol 26: 693-701, 2009.

10. Metz TD, Stoddard GJ, Henry E, Jackson M, Holmgren C and Esplin S: Simple, validated vaginal birth after cesarean delivery prediction model for use at the time of admission. Obstet Gynecol 122: 571-578, 2013.

11. Harris BS, Heine RP, Park J, Faurot KR, Hopkins MK, Rivara AJ, Kemeny HR, Grotegut CA and Jelovsek JE: Are prediction models for vaginal birth after cesarean accurate? Am J Obstet Gynecol 220: 492, 2019. 
12. Cahill AG, Stamilio DM, Odibo AO, Peipert JF, Ratcliffe SJ, Stevens EJ, Sammel MD and Macones GA: Is vaginal birth after cesarean (VBAC) or elective repeat cesarean safer in women with a prior vaginal delivery? Am J Obstet Gynecol 195: 1143-1147, 2006.

13. Grobman WA, Lai Y, Landon MB, Spong CY, Leveno KJ, Rouse DJ, Varner MW, Moawad AH, Caritis SN, Harper M, et al: Eunice kennedy shriver national institute of child health and human development maternal-fetal medicine units network: Can a prediction model for vaginal birth after cesarean also predict the probability of morbidity related to a trial of labor? Am J Obstet Gynecol 200: 56, 2009.

14. Lipschuetz M, Guedalia J, Rottenstreich A, Persky MN, Cohen SM, Kabiri D, Levin G, Yagel S, Unger R and Sompolinsky Y: Prediction of vaginal birth after cesarean deliveries using machine learning. Am J Obstet Gynecol 222: 613, 2020.

15. Witteman CL, Renooij S and Koele P: Medicine in words and numbers: A cross-sectional survey comparing probability assessment scales. BMC Med Inform Decis Mak 7: 13, 2007.

16. Al-Zirqi I and Vangen S: Prelabour uterine rupture: Characteristics and outcomes. BJOG 127: 1637-1644, 2020.

17. Cecchini F, Tassi A, Londero AP, Baccarini G, Driul L and Xodo S: First trimester uterine rupture: A case report and literature review. Int J Environ Res Public Health 17: 2976, 2020.

18. Bradley LM, Addas JAK and Herath JC: Maternal and fetal death at 22 weeks following uterine rupture at the site of the placenta percreta in a C-section scar. Forensic Sci Med Pathol 15 658-662, 2019.

19. Martel MJ and MacKinnon CJ: No. 155-guidelines for vaginal birth after previous caesarean birth. J Obstet Gynaecol Can 40 e195-e207, 2018.

20. Sarwar I, Akram F, Khan A, Malik S, Islam A and Khan K: Validity of transabdominal ultrasound scan in the prediction of uterine scar thickness. J Ayub Med Coll Abbottabad 32: 68-72, 2020.

21. Cegolon L, Mastrangelo G, Maso G, Dal Pozzo G, Ronfani L, Cegolon A, Heymann WC and Barbone F: Understanding factors leading to primary cesarean section and vaginal birth after cesarean delivery in the friuli-venezia giulia region (North-Eastern Italy), 2005-2015. Sci Rep 10: 380, 2020.

22. Lehmann S, Baghestan E, Børdahl PE, Irgens LM and Rasmussen S: Low risk pregnancies after a cesarean section: Determinants of trial of labor and its failure. PLoS One 15 : e0226894, 2020

23. Tenore JL: Methods for cervical ripening and induction of labor. Am Fam Physician 67: 2123-2128, 2003.

24. Dy J, DeMeester S, Lipworth H and Barrett J: No. 382-trial of labour after caesarean. J Obstet Gynaecol Can 41: 992-1011, 2019. Erratum in: J Obstet Gynaecol Can 42: 1452, 2020.

25. Wingert A, Hartling L, Sebastianski M, Johnson C Featherstone R, Vandermeer B and Wilson RD: Clinical interventions that influence vaginal birth after cesarean delivery rates: Systematic review \& meta-analysis. BMC Pregnancy Childbirth 19: 529, 2019.

26. Catling-Paull C, Johnston R, Ryan C, Foureur MJ and Homer CS Clinical interventions that increase the uptake and success of vaginal birth after caesarean section: A systematic review. J Adv Nurs 67: 1646-1661, 2011.

27. West HM, Jozwiak M and Dodd JM: Methods of term labour induction for women with a previous caesarean section. Cochrane Database Syst Rev 6: CD009792, 2017.

28. Yap OW, Kim ES and Laros RK Jr: Maternal and neonatal outcomes after uterine rupture in labor. Am J Obstet Gynecol 184: $1576-1581,2001$

29. Leung AS, Farmer RM, Leung EK, Medearis AL and Paul RH: Risk factors associated with uterine rupture during trial of labor after cesarean delivery: A case-control study. Am J Obstet Gynecol 168: 1358-1363, 1993.

30. Ridgeway JJ, Weyrich DL and Benedetti TJ: Fetal heart rate changes associated with uterine rupture. Obstet Gynecol 103 506-512, 2004.
31. Guiliano M, Closset E, Therby D, LeGoueff F, Deruelle P and Subtil D: Signs, symptoms and complications of complete and partial uterine ruptures during pregnancy and delivery. Eur J Obstet Gynecol Reprod Biol 179: 130-134, 2014.

32. Walsh CA and Baxi LV: Rupture of the primigravid uterus: A review of the literature. Obstet Gynecol Surv 62: 327-334, 2007.

33. Attarde VY, Patil P, Chaudhari R, Zope N and Apte A: Sonographic findings of uterine rupture with expulsion of the fetus into broad ligament. J Clin Ultrasound 37: 50-52, 2009.

34. Ozdemir I, Yucel N and Yucel O: Rupture of the pregnant uterus: A 9-year review. Arch Gynecol Obstet 272: 229-231, 2005.

35. Chibber R, El-Saleh E, Al Fadhi R, Al Jassar W and Al Harmi A: Uterine rupture and subsequent pregnancy outcome-how safe is it? A 25-year study. J Matern Fetal Neonatal Med 23: 421-424, 2010.

36. Trojano G, Damiani GR, Olivieri C, Villa M, Malvasi A, Alfonso R, Loverro M and Cicinelli E: VBAC: Antenatal predictors of success. Acta Biomed 90: 300-309, 2019.

37. Flamm BL, Fried MW, Lonky NM and Giles WS: External cephalic version after previous cesarean section. Am J Obstet Gynecol 165: 370-372, 1991.

38. Clock C, Kurtzman J, White J and Chung JH: Cesarean risk after successful external cephalic version: A matched, retrospective analysis. J Perinatol 29: 96-100, 2009.

39. Sela HY, Fiegenberg T, Ben-Meir A, Elchalal U and Ezra Y: Safety and efficacy of external cephalic version for women with a previous cesarean delivery. Eur J Obstet Gynecol Reprod Biol 142: 111-114, 2009.

40. Impey ORE, Greenwood CEL and Impey LWM: External cephalic version after previous cesarean section: A cohort study of 100 consecutive attempts. Eur J Obstet Gynecol Reprod Biol 231: 210-213, 2018.

41. urcan N, Bohiltea RE, Ionita-Radu F, Furtunescu F, Navolan D, Berceanu C, Nemescu D and Cirstoiu MM: Unfavorable influence of prematurity on the neonatal prognostic of small for gestational age fetuses. Exp Ther Med 20: 2415-2422, 2020.

42. Bohîlțea R, Furtunescu F, Turcan N, Navolan D, Ducu I and Cîrstoiu M: Prematurity and intrauterine growth restriction: Comparative analysis of incidence and short term complication. Proceedings of SOGR 2018. In: Proceedings of the 17th National Congress of The Romanian Society of Obstetrics and Gynecology 2018, pp708-712, 2019.

43. Kaya GK, Ward JR and Clarkson PJ: A framework to support risk assessment in hospitals. Int J Qual Health Care 31: 393-401, 2019.

44. Thompson S, Neal S and Clark V: Clinical risk management in obstetrics: Eclampsia drills. BMJ 328: 269-271, 2004.

45. Klein VR: Risk management in obstetrics and gynecology. Clin Obstet Gynecol 62: 550-559, 2019.

46. Romano AM, Gerber H and Andrews D: Social media, power, and the future of VBAC. J Perinat Educ 19: 43-52, 2010.

47. Keedle H, Peters L, Schmied V, Burns E, Keedle W and Dahlen HG: Women's experiences of planning a vaginal birth after caesarean in different models of maternity care in Australia. BMC Pregnancy Childbirth 20: 381, 2020.

48. Poprzeczny AJ, Stocking K, Showell M and Duffy JMN: Patient decision aids to facilitate shared decision making in obstetrics and gynecology: A systematic review and meta-analysis. Obstet Gynecol 135: 444-451, 2020.

49. Faiz AS and Ananth CV: Etiology and risk factors for placenta previa: An overview and meta-analysis of observational studies. J Matern Fetal Neonatal Med 13: 175-190, 2003.

50. Cresswell JA, Ronsmans C, Calvert C and Filippi V: Prevalence of placenta praevia by world region: A systematic review and meta-analysis. Trop Med Int Health 18: 712-724, 2013. International (CC BY-NC-ND 4.0) License. 